\title{
GERAÇÃO DE PADRÕES ÓTIMOS DE CORTE ATRAVÉS DE ALGORITMOS DE TRAÇAMENTO APLICADOS A FUSTES INDIVIDUAIS ${ }^{1}$
}

\author{
Julio Eduardo Arce 2 , Patricio MacDonagh ${ }^{3}$ e Ramón Alejandro Friedl ${ }^{4}$
}

\begin{abstract}
RESUMO - No presente trabalho descreve-se uma metodologia de otimização do corte ou traçamento de árvores para obtenção de multiprodutos em nível de fustes individuais. A geração dos padrões ótimos de corte foi realizada através de dois módulos: 1) um algoritmo heurístico que identifica os cortes potenciais (números úteis), que devem ser avaliados ao longo do fuste; e 2) um algoritmo com base na programação dinâmica (PD), que determina a combinação ótima de toras de cada tipo de comprimento a serem retiradas do fuste, visando maximizar o seu comprimento utilizado. Um exemplo da obtenção dos padrões ótimos de corte para um conjunto de 25 fustes é apresentado. $\mathrm{O}$ traçamento ótimo e o traçamento real obtido pelo motosserrista foram comparados. O resíduo de madeira deixado na floresta foi reduzido de 16 para $5 \%$ através dos padrões de corte ótimos.
\end{abstract}

Palavras-chave: Otimização, programação dinâmica, heurísticas e Pinus.

\section{OPTIMAL BUCKING PATTERN GENERATION THROUGH CUTTING ALGORITHMS APPLIED TO INDIVIDUAL STEMS}

\begin{abstract}
This work describes a method of optimizing individual tree stem bucking operations. The individual optimal cutting patterns were generated by means of two modules: 1) a heuristic algorithm which identifies the potential cuts (useful numbers) to be evaluated along the stem, and 2) a Dynamic Programming based-algorithm to determine the optimum combination of products to be obtained to maximize stem profit. An example is provided to illustrate the optimum cutting patterns for 25 individual stems. Optimal and conventional bucking patterns were compared. Residues were reduced from about $16 \%$ to $5 \%$ by means of the optimal bucking patterns.
\end{abstract}

Key words: Optimization, dynamic programming, heuristics and Pinus.

\section{INTRODUÇÃO}

A colheita representa a operação final de um ciclo de produção florestal, na qual são obtidos os produtos mais valiosos, constituindo um dos fatores que determinam a rentabilidade florestal. A maneira como é conduzido o traçamento das árvores obtidas na colheita ou corte raso é fundamental para estabelecer as quantidades de cada um dos diferentes tipos de produtos que podem ser obtidos da floresta. Porém, esta tarefa é executada quase exclusivamente pelo motosserrista, com base na sua intuição, o que pode comprometer a rentabilidade do negócio florestal.

O traçamento das árvores derrubadas nas operações de colheita florestal pode ser considerado como um processo de tomada de decisões de curto prazo. De fato, a posição dos cortes que definirão as toras deve ser

Recebido para publicação em 4.4.2002 e aceito para publicação em 30.4.2004.

Departamento de Ciências Florestais, Universidade Federal do Paraná, Av. Pref. Lothário Meissner, 3400, 80210-170 CuritibaPR, Tel.: +55-41-360-4213 - Fax: +55-41-360-4231, <jarce@ floresta.ufpr.br>. ${ }^{3}$ Cátedra de Aprovechamiento Forestal, Fac.de Ciencias Forestales, Universidad Nacional de Misiones, Bertoni 124, Caixa Postal 3380, Eldorado, Misiones, Argentina, Tel.: +543751-431526, <mdonagh @ facfor.unam.edu.ar>; ${ }^{4}$ Cátedra de Dasometría, Fac. de Ciencias Forestales, Universidad Nacional de Misiones, <afriedl@altoparana.com>. 
decidida no local, após a visualização da árvore abatida, e esta tarefa muitas vezes é realizada sem o suporte de ferramentas de otimização. Quando um único comprimento de tora é retirado da floresta estas decisões tornamse bem mais simples, porém no caso de multiprodutos florestais a complexidade combinatória necessária para atingir o traçamento otimizado aumenta. A utilização de técnicas de otimização aplicada à obtenção de multiprodutos florestais pode contribuir de forma significativa para aumentar a utilização da matéria-prima, reduzindo os resíduos deixados na floresta ou subutilizados, ao serem aproveitados como energia.

Em algumas indústrias, como as de vidro, metalúrgica e têxtil, o planejamento da produção depara-se freqüentemente com os denominados problemas de corte. Nestas indústrias, a redução dos custos de produção é freqüentemente obtida através da seguinte estratégia: a matéria-prima utilizada é inicialmente produzida em tamanhos grandes padronizados, possivelmente estocada e, somente mais tarde, reduzida a tamanhos menores, para então ser utilizada pela indústria, ou para atender às demandas externas de tamanhos variados, muitas vezes não-padronizados (Arenales \& Morabito, 1997). Esta estratégia gera a necessidade de um estágio adicional de produção, denominado corte ou desdobramento da matéria-prima. As perdas decorrentes destas operações de corte são inevitáveis, motivando o seu planejamento para minimizar os efeitos negativos gerados pelo desperdício sobre os custos de produção.

Sob essa mesma ótica, as florestas comerciais podem ser vistas como fábricas produtoras de grandes peças de matéria-prima - árvores - de dimensões conhecidas distribuições diamétricas e hipsométricas - e com uma grande capacidade de estoque. De fato, por ser a árvore fábrica e produto ao mesmo tempo (Gregersen \& Contreras, 1980), a totalidade da produção da floresta vai sendo estocada nas árvores até o momento da colheita, quando elas devem ser derrubadas e cortadas em peças menores - toras -, atendendo aos diversos pedidos realizados pelos clientes.

Alguns sistemas de inventário florestal e prognose do crescimento e da produção existentes no mercado possuem módulos de sortimento que informam a quantidade de cada tipo de produto que o talhão possui. Mas para tal fim, estes sistemas consideram somente a otimização da floresta em pé - otimização em função da oferta -, uma vez que não dispõem de cadastros de pedidos ou demandas mínimas por tipo de produto. Dentre estes sistemas podem ser mencionados o sistema de inventário MARVL (Method for assessment of recoverable volume by log types), desenvolvido na Nova Zelândia a partir do trabalho de Deadman \& Goulding (1978), e o SISPINUS (Oliveira, 1995), desenvolvido especificamente pela EMBRAPACNPF (Empresa Brasileira de Pesquisa Agropecuária Centro Nacional de Pesquisas de Florestas) para plantios de Pinus spp. no Sul do Brasil, os quais não são alimentados com informações de demanda.

No trabalho pioneiro de Borges (1981) foi desenvolvido, para Pinus taeda L., no Estado do Paraná, Brasil, um programa de computação para o seccionamento do fuste, visando obter, de cada árvore, o número máximo de peças serradas com as maiores dimensões. Para tal fim, foram construídas funções de afilamento-padrão relativas por classes do fator de forma natural, baseadas na função polinomial de quinto grau. A partir dos mesmos dados utilizados pelo autor, foram utilizadas curvas de afilamento em séries de quocientes naturais transformados. O objetivo foi estimar volumes parciais através da integração, entre os pontos relativos desejados, das equações que descrevem estas séries (Jorge, 1982).

Dentre os trabalhos desenvolvidos no Brasil, foi apresentado em 1998, por Andrade \& Leite, um método para quantificar multiprodutos de árvores individuais na unidade estere. Em Assis et al. (2001), encontra-se uma comparação entre modelos polinomiais segmentados e não-segmentados na estimativa de diâmetros e volumes para Pinus taeda. Foram comparados neste trabalho o modelo segmentado de Clark et al., o modelo segmentado de Max e Burkhart, o polinômio de potências fracionárias e inteiras de Hradetzky e o modelo de Goulding e Murray.

No trabalho de Faaland \& Briggs (1984), foi utilizada a PD para resolver o problema do corte ótimo das árvores em produtos serrados terminados. Este problema se caracteriza por duas etapas: o desdobramento da árvore em toras e a subseqüente transformação destas toras em produtos como tábuas, sarrafos e demais peças obtidas no desdobro. Estas duas atividades foram consideradas como um sistema único de produção.

Uma abordagem de subalgoritmos hierárquicos de PD foi utilizada por Reinders \& Hendriks (1989), para conversão ótima de árvores em peças serradas. Esta conversão foi descrita em três níveis: 1) árvores em toras; 2) toras em peças - fatias -, obtidas por cortes paralelos realizados nas toras; e 3) peças menores, obtidas por cortes paralelos entre si e perpendiculares aos cortes anteriores.

\section{R. Árvore, Viçosa-MG, v.28, n.2, p.207-217, 2004}


Maness \& Adams (1991) utilizaram um modelo estruturado como um problema de PL. O modelo utilizou estratégias de traçamento de árvores em toras e de toras em peças serradas, através de um procedimento de resolução de três estágios. O volume dos produtos elaborados foi controlado através de relações preço-volume, simulando curvas de demanda.

O sistema OPTICORT, desenvolvido pela equipe chilena de pesquisadores liderada pelo professor Weintraub, para um grupo de empresas florestais do Chile (Epstein et al., 1999), faz parte de uma verdadeira malha de sistemas de Pesquisa Operacional aplicados ao planejamento e desenvolvimento do setor florestal. A malha está formada por um sistema de planejamento florestal de longo prazo (PLANEX), um sistema de otimização do sortimento (OPTICORT) e um sistema de programação do transporte florestal (ASICAM). Em particular, o OPTICORT consiste em um módulo de PL combinado com um algoritmo de geração de colunas. Este algoritmo, na verdade, é um gerador de padrões de corte tipo branchand-bound, acrescentando, a partir da ramificação e avaliação iterativa de uma árvore de alternativas, opções às soluções ótimas encontradas pelo módulo de PL.

O objetivo deste trabalho foi descrever uma metodologia para otimizar o traçamento de fustes de árvores florestais, baseada em um algoritmo heurístico e na programação dinâmica, que se valora através da quantificação das perdas de madeira para serraria ou pasta celulósica, obtidas em campo pelos métodos tradicionais.

\section{MATERIAL EMÉTODOS}

Para avaliar a eficácia da metodologia proposta, foi rigorosamente quantificada uma operação real de colheita e traçamento, utilizando 25 fustes de Pinus spp., selecionados de forma aleatória. Os dados foram obtidos na província de Misiones, Argentina. Para cada fuste, foi obtido o comprimento útil da porção utilizável para serraria, considerando um diâmetro comercial de $18 \mathrm{~cm}$. No Quadro 1 estão as características dos fustes utilizados.

Após a medição do comprimento útil de todos os fustes selecionados, foi executado o traçamento por um motosserrista idôneo, sem a presença da equipe de medição, segundo o critério-padrão, ou seja, da maneira como habitualmente é feito. É importante frisar que o operário desconhecia a avaliação que estava sendo realizada. Os comprimentos de tora aceitos pela serraria foram $l_{1}=12$ ' (pés), $l_{2}=14^{\prime}$ e $l_{3}=16^{\prime}$. Entretanto, na prática, ao converter estes comprimentos ao sistema métrico decimal e incluir a folga para a destopa na serraria, estes três comprimentos passam a ser $l_{1}=3,75 \mathrm{~m}, l_{2}=4,35 \mathrm{~m}, l_{3}=4,99 \mathrm{~m}$.

Após o traçamento foram medidas, em cada um dos fustes, todas as toras que o motosserrista obteve, caracterizando, desta maneira, a situação ou o cenário real de traçamento.

A situação otimizada de traçamento foi obtida através de padrões de corte preestabelecidos. Para tanto, inicialmente foram definidos alguns volumes e resíduos em nível de fustes individuais, conforme mostrado na Figura 1.

A geração dos padrões de corte ótimos foi efetivada através de dois módulos: 1) um algoritmo heurístico que identifica os cortes potenciais (números úteis), que devem ser avaliados ao longo do fuste, e 2) um algoritmo com base na programação dinâmica (PD) que determina a combinação ótima de produtos a serem retirados do fuste, com o objetivo de maximizar o seu valor (Gilmore \& Gomory, 1961, 1963, 1965; Carnieri et al., 1994a, 1994b).

Quadro 1 - Características da amostra de fustes utilizada para testar os algoritmos Table 1 - Characteristics of the stem sample used to test the algorithms

\begin{tabular}{|c|c|c|c|c|c|c|c|c|c|}
\hline Fuste & $\mathrm{L}^{\underline{1}}+$ & Fuste & $\mathrm{L}$ & Fuste & $\mathrm{L}$ & Fuste & $\mathrm{L}$ & Fuste & L \\
\hline 1 & 3,90 & 6 & 7,40 & 11 & 10,80 & 16 & 13,86 & 21 & 16,43 \\
\hline 2 & 5,50 & 7 & 9,00 & 12 & 11,50 & 17 & 13,95 & 22 & 16,90 \\
\hline 3 & 6,80 & 8 & 9,03 & 13 & 11,85 & 18 & 14,30 & 23 & 17,32 \\
\hline 4 & 6,85 & 9 & 10,53 & 14 & 11,90 & 19 & 15,21 & 24 & 18,15 \\
\hline 5 & 7,30 & 10 & 10,77 & 15 & 12,81 & 20 & 16,12 & 25 & 18,32 \\
\hline
\end{tabular}

${ }^{1 /} \mathrm{L}$ : comprimento útil medido até um diâmetro comercial com casca de $18 \mathrm{~cm}$. 


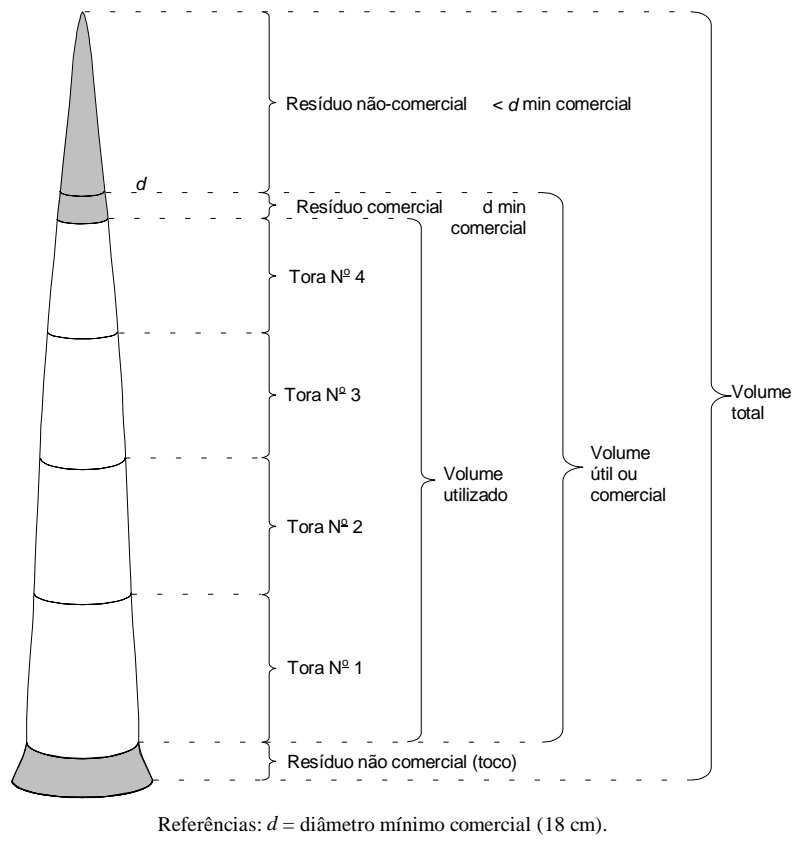

Figura 1 - Diferentes volumes e resíduos identificados nos fustes individuais.

Figure 1 -Different volumes and residues identified in the individual stems.

\subsection{Algoritmo Heurístico para Geração dos Números Úteis (Algoritmo 1)}

O algoritmo heurístico para geração dos números úteis foi adaptado a partir da proposta de Carnieri et al. (1994a), em seu trabalho de otimização do corte bidimensional de painéis de madeira compensada. Na seqüência descreve-se o algoritmo proposto para geração do conjunto útil de pontos onde serão avaliadas as estratégias de corte ao longo do fuste de comprimento $L$, considerando $m$ produtos de comprimentos diferentes $l_{1}, l_{2}, \ldots, l_{m}$.

Inicialmente é obtido o menor comprimento $\beta$ a partir da lista de diferentes produtos; é definido também o primeiro número útil como sendo o início do comprimento útil (Passo 1). Na sequiência, a partir de cada número útil encontrado, devem ser calculados os pontos de corte ao longo do fuste para cada novo produto $k$ extraindo $j$ toras $(j=1,2, \ldots)$, desde que a porção remanescente do fuste seja suficientemente longa para retirar pelo menos uma tora do produto de menor comprimento $\beta$ (Passo 2). Os números úteis encontrados desta forma $-F^{(k)}$ - são acrescentados na lista existente $-U^{(k+1)}-$, removendo-se quaisquer números repetidos ou equivalentes (Passo 3). Finalmente, o procedimento se repete até considerar o último produto $(k=1)$, concluindo a geração dos números úteis (Passo 4), os quais são armazenados no vetor $U^{(1)}$. A Figura 2 apresenta o pseudocódigo deste algoritmo.

\subsection{Algoritmo de PD para Geração do Padrão de Corte Ótimo (Algoritmo 2)}

A programação dinâmica $(\mathrm{PD})$, também denominada programação em múltiplos estágios, caracteriza-se pela avaliação sucessiva da evolução da otimização em estágios através de uma equação recursiva. Em cada um destes estágios são comparados vários estados ou alternativas, e é guardado somente o valor - label - do melhor destes. Desta maneira, conforme a proposta de Gilmore \& Gomory (1961) e Carnieri et al. (1994a), os diferentes

Passo 1. Calcule $=\min \left\{l_{i}, i=1,2, \ldots, m\right\}$. Defina $U^{(m+1)}=F^{(m+1)}=\{0\}$, e faça $k=m$.

Passo 2. Calcule $F^{(k)}=\left\{u+j * l_{k} ; u \quad U^{(k+1)}, j=1,2, \ldots\right.$, e $\left.L-\left(u+j * l_{k}\right) \quad\right\}$

Passo 3. Faça $U^{(k)}=F^{(k)} \quad U^{(k+1)}$. Elimine quaisquer números equivalentes de $U^{(k)}$.

Passo 4. Se $k>1$, faça $k \quad k-1$ e vá para o Passo 2. Caso contrário, pare. $U^{(1)}$ contém a lista com os números úteis.

em que $\quad L=$ comprimento útil do fuste;

$m$ = número de produtos considerados;

$l_{1}, l_{2}, \ldots, l_{m}=$ comprimentos individuais de cada um dos produtos;

$=$ menor dos comprimentos dos $m$ produtos considerados;

$F ; U=$ vetores com os números úteis gerados em cada etapa.

Figura 2 - Pseudocódigo do algoritmo heurístico de geração dos números úteis.

Figure 2 - Pseudo code of the heuristic algorithm generating useful numbers. 
produtos que são considerados na otimização de um determinado fuste ou classe diamétrica representam as etapas do problema de PD, e os números úteis gerados através do algoritmo constituem os estados. A seguinte equação recursiva tem por função maximizar o valor do fuste.

$\max F_{s}(x)=\max \left\{P_{s}+F_{s}\left(x-l_{s}\right), F_{s-1}(x)\right\} \quad s=2,3, \ldots, m$

em que $x=$ ponto do fuste no qual é realizada a avaliação; $F_{s}(x)=$ valor da melhor combinação de produtos obtida até o comprimento $x$, utilizando somente os primeiros $s$ produtos; $l_{s}=$ comprimento do produto $s ; P_{s}=$ valor da tora do produto $s$ que está sendo considerado; e $F_{s}(x-l)$ = valor da melhor combinação de produtos obtida até o comprimento $\left(x-l_{s}\right)$, utilizando somente os primeiros $s$ produtos.

\subsection{Padrões Ótimos de Corte}

A partir dos dois algoritmos descritos nas seções anteriores foram gerados os padrões ótimos de corte para os fustes analisados. Para cada fuste foram gerados os números úteis, que indicam os cortes potenciais que poderiam ser realizados, através do algoritmo 1. A partir destes números úteis, foi posteriormente gerado o padrão de corte ótimo, através do algoritmo 2.

\subsection{Comparação Entre as Operações de Traçamento Real e Otimizado}

Finalmente, foram comparados o traçamento real e o traçamento otimizado, como forma de validar a metodologia proposta. Para realizar esta comparação, foi utilizada a diferença agregada, de modo a quantificar a diferença total ou cumulativa entre as operações de traçamento real e otimizado.

\section{RESULTADOS E DISCUSSÃO}

\subsection{Geração dos Números Úteis}

A geração dos números úteis é ilustrada através do seguinte exemplo. Seja um fuste de comprimento útil $L=$ $18,35 \mathrm{~m}$ até um diâmetro comercial de $18 \mathrm{~cm}$. Os $m=3$ produtos diferentes a serem obtidos devem possuir, respectivamente, os seguintes comprimentos: $l_{1}=3,75 \mathrm{~m}$, $l_{2}=4,35 \mathrm{~m}, l_{3}=4,99 \mathrm{~m}$. Ao realizar uma busca exaustiva, considerando, por exemplo, o máximo divisor comum dos três comprimentos de tora, que neste caso é $1 \mathrm{~cm}$, surgem
$(18,35 \mathrm{~m} / 0,01 \mathrm{~m})=1.835$ pontos $(0,01 ; 0,02 ; 0,03 ; \ldots ; 18,33$; 18,34 ; e 18,35). Através do algoritmo proposto foram obtidos 19 pontos, o que representa uma redução considerável no número de pontos a avaliar. Estes pontos, que representam possíveis cortes ao longo do fuste do exemplo, são 3,$75 ; 4,35 ; 4,99 ; 7,50 ; 8,10 ; 8,70 ; 8,74 ; 9,34$; 9,$98 ; 11,25 ; 11,85 ; 12,45 ; 12,49 ; 13,05 ; 13,09 ; 13,69 ; 13,73$; 14,33; e 18,35. O último valor $(18,35)$ não é obtido a partir do pseudocódigo apresentado; seu ingresso é forçado para poder quantificar, posteriormente, as perdas de aproveitamento no traçamento do fuste.

Durante a geração dos números úteis não é estabelecida a ordem em que as diferentes toras poderiam ser obtidas do fuste; este ordenamento é realizado posteriormente, na geração dos padrões de corte.

\subsection{Obtenção do Padrão Ótimo de Corte Através do Algoritmo de PD}

No Quadro 2 está o procedimento de solução através do algoritmo de PD proposto, para o mesmo fuste de 18,35 m utilizado como exemplo na geração dos números úteis.

Em um problema de PD a solução ótima geralmente deve ser reconstruída a partir da equação recursiva. Este procedimento, denominado de reconstrução da trajetória, é indicado no Quadro 2 através das células hachuradas. O valor ótimo encontrado aparece na célula inferior direita da última linha do Quadro 2, correspondente ao último estado $(x=18,35)$ no último estágio $(s=3)$. O valor desta célula $(18,08 \mathrm{~m})$ indica que traçando o fuste de forma ótima são utilizados 18,08 m. O resíduo associado a esta solução ótima é de $0,27 \mathrm{~m}$, obtido através da diferença entre o comprimento útil (18,35 m) e o comprimento efetivamente utilizado (18,08 m). A partir deste vértice começa a reconstrução da trajetória. O último produto retirado na tora que gera o valor ótimo aparece sobrescrito na célula. Neste caso, o número 3 indica que a última tora retirada é do produto 3 , com um comprimento $l_{3}$ de 4,99 m. Portanto, a célula que segue na reconstrução da trajetória é aquela localizada no estado $x=13,09 \mathrm{~m}$, subtraindo o comprimento da tora $\left(l_{3}=4,99 \mathrm{~m}\right)$ do valor ótimo obtido no último estado (18,08 m), ou seja, analisando a porção remanescente do fuste após a retirada de uma tora de 4,99 m. Este comprimento remanescente corresponde ao estado $x=$ $13,09 \mathrm{~m}$.

A célula que segue na reconstrução da trajetória localiza-se no estágio $s=3$, estado $x=13,09$. Nesta célula 
aparece novamente o número 3 (hachurado), indicando que a penúltima tora do padrão ótimo de corte também é do produto 3, cujo comprimento é de 4,99 $\mathrm{m}$.

Quadro 2 - Exemplo do algoritmo de PD para geração do padrão ótimo de corte, considerando um fuste de comprimento útil $L=18,35 \mathrm{~m}$ e três comprimentos de tora diferentes $\left(l_{1}=3,75 \mathrm{~m} ; l_{2}=4,35 \mathrm{~m} ; l_{3}=4,99 \mathrm{~m}\right)$. Células hachuradas indicam a reconstrução da trajetória para encontrar a solução ótima

Table 2 - Example of the bucking-pattern generation DP algorithm considering a stem with useful length $L=$ $18,35 \mathrm{~m}$ and three different log lengths $\left(l_{1}=3,75 \mathrm{~m} ; l_{2}=\right.$ $4,35 \mathrm{~m} ; l_{3}=4,99 \mathrm{~m}$ ). Grayed cells indicate the optimal solution trajectory recovery

\begin{tabular}{|c|c|c|c|c|}
\hline \multirow{3}{*}{ Estado $^{1 /}$} & \multirow{2}{*}{$x$} & \multicolumn{3}{|c|}{ Estágio $^{2 /}$} \\
\hline & & $s=1$ & $s=2$ & $s=3$ \\
\hline & (m) & \multicolumn{3}{|c|}{ (m) } \\
\hline 1 & 3,75 & $3,75^{l}$ & $3,75^{1}$ & $3,75^{1}$ \\
\hline 2 & 4,35 & $3,75^{1}$ & $4,35^{2}$ & $4,35^{2}$ \\
\hline 3 & 4,99 & $3,75^{1}$ & $4,35^{2}$ & $4,99^{3}$ \\
\hline 4 & 7,50 & $7,50^{1}$ & $7,50^{1}$ & $7,50^{1}$ \\
\hline 5 & 8,10 & $7,50^{1}$ & $8,10^{2}$ & $8,10^{2}$ \\
\hline 6 & 8,70 & $7,50^{1}$ & $8,70^{2}$ & $8,70^{2}$ \\
\hline 7 & 8,74 & $7,50^{1}$ & $8,70^{2}$ & $8,74^{3}$ \\
\hline 8 & 9,34 & $7,50^{1}$ & $8,70^{2}$ & $9,34^{3}$ \\
\hline 9 & 9,98 & $7,50^{1}$ & $8,70^{2}$ & $9,98^{3}$ \\
\hline 10 & 11,25 & $11,25^{1}$ & $11,25^{1}$ & $11,25^{\prime}$ \\
\hline 11 & 11,85 & $11,25^{1}$ & $11,85^{2}$ & $11,85^{2}$ \\
\hline 12 & 12,45 & $11,25^{1}$ & $11,85^{2}$ & $9,98^{3}$ \\
\hline 13 & 12,49 & $11,25^{1}$ & $12,45^{2}$ & $12,49^{3}$ \\
\hline 14 & 13,05 & $11,25^{1}$ & $13,05^{2}$ & $13,05^{2}$ \\
\hline 15 & 13,09 & $11,25^{1}$ & $13,05^{2}$ & $13,09^{3}$ \\
\hline 16 & 13,69 & $11,25^{1}$ & $13,05^{2}$ & $13,69^{3}$ \\
\hline 17 & 13,73 & $11,25^{1}$ & $13,05^{2}$ & $13,73^{3}$ \\
\hline 18 & 14,33 & $11,25^{1}$ & $13,05^{2}$ & $14,33^{3}$ \\
\hline 19 & 18,35 & $15,00^{1}$ & $17,40^{2}$ & $18,08^{3}$ \\
\hline
\end{tabular}

1/ Os estados correspondem aos números úteis gerados pelo algoritmo heurístico.

2` O sobrescrito em itálico indica o último produto retirado ao otimizar o estado $x$ do estágio $s$.

$\mathrm{Na}$ seqüência, prosseguindo com a reconstrução da trajetória, é obtida a célula correspondente ao estado $x=8,10 \mathrm{~m}$, subtraindo o comprimento da tora anterior $(4,99 \mathrm{~m})$ ao estado anterior $(13,09 \mathrm{~m}-4,99 \mathrm{~m}=8,10 \mathrm{~m})$. Neste caso, a célula hachurada com o número sobrescrito 2 , correspondente ao estado $x=8,10 \mathrm{~m}$ e ao estágio $s=3$, indica que a antepenúltima tora a ser retirada é do produto
2 , cujo comprimento é de $4,35 \mathrm{~m}$. Deste modo, mantendo o estado $x=8,10 \mathrm{~m}$, a célula hachurada se desloca para a coluna da esquerda, correspondente ao estágio $s=2$.

Finalmente, a partir da célula anterior é subtraído o comprimento da tora anterior $\left(l_{2}=4,35\right)$ ao estado anterior $x=8,10 \mathrm{~m}$. O valor obtido de $3,75 \mathrm{~m}$ indica o estado no qual deve se localizar a tora seguinte no padrão de corte ótimo. Observa-se no Quadro 2 que a célula hachurada correspondente ao estado $x=3,75 \mathrm{~m}$ no estágio $s=2$ indica que a tora seguinte é do produto $1\left(l_{1}=3,75 \mathrm{~m}\right)$.

Em síntese, a partir da reconstrução da trajetória é obtido o padrão de corte ótimo para o fuste utilizado como exemplo, que consiste em retirar uma tora de $3,75 \mathrm{~m}$, uma tora de $4,35 \mathrm{~m}$ e duas toras de $4,99 \mathrm{~m}$, totalizando $3,75+4,35+2 \times 4,99=18,08 \mathrm{~m}$.

\subsection{Implementação dos Padrões Ótimos de Corte Através de Regras de Traçamento}

A implementação prática dos resultados deste trabalho pode ser feita, por exemplo, através da elaboração de regras de traçamento, as quais seriam distribuídas de forma impressa e plastificadas entre os motosserristas. Estas regras deveriam ser atualizadas cada vez que algum novo sortimento com diâmetro e, ou, comprimento diferente for demandado por parte dos compradores das toras. O Quadro 3 contém os padrões de corte ótimos, gerados a partir dos algoritmos 1 e 2 , considerando três comprimentos diferentes de tora $\left(l_{1}=3,75 \mathrm{~m} ; l_{2}=4,35 \mathrm{~m}\right.$; $l_{3}=4,99 \mathrm{~m}$ ), para fustes com comprimentos úteis teóricos de 4,00 a 24,00 m, com intervalos de $50 \mathrm{~cm}$. Somente por uma questão de espaço não se apresentam os padrões de corte para fustes com intervalos menores, por exemplo 10,5 e $1 \mathrm{~cm}$. Porém, para validar a metodologia proposta, os padrões de corte foram gerados para fustes com intervalos de $1 \mathrm{~cm}$ no que se refere ao comprimento útil. Estes padrões de corte constam na base de dados do programa utilizado para geração dos padrões ótimos de corte.

No exemplo considerado, foi analisado um único tipo de produto com seu diâmetro comercial $(d)$, o que significa que, analogamente, apenas um único comprimento útil foi considerado em cada fuste. Para o caso de múltiplos $d$ 's, simplesmente deveriam ser utilizados os algoritmos $1 \mathrm{e} 2 \mathrm{em}$ forma repetitiva, tantas vezes quanto diferentes $d$ devam ser avaliados, começando pelas toras de maior $d$ e finalizando com as de menor $d$. Ao comprimento útil do fuste correspondente a cada $d$ deve ser

\section{R. Árvore, Viçosa-MG, v.28, n.2, p.207-217, 2004}


Quadro 3 - Padrões ótimos de corte gerados, considerando três diferentes comprimentos de tora $\left(l_{1}=3,75 \mathrm{~m} ; l_{2}=4,35 \mathrm{~m} ; l_{3}\right.$ $=4,99 \mathrm{~m}$ ) para fustes com comprimentos úteis teóricos de 4,00 a $24,00 \mathrm{~m}$, com intervalos de $50 \mathrm{~cm}$

Table 3 - Optimal bucking-pattern generated considering three different log lengths $\left(l_{1}=3,75 \mathrm{~m} ; l_{2}=4,35 \mathrm{~m} ; l_{3}=4,99 \mathrm{~m}\right)$ for stems with theoretical useful lengths varying from 4,00 to $24,00 \mathrm{~m}$, with a $50 \mathrm{~cm}$ interval

\begin{tabular}{|c|c|c|c|c|c|c|c|}
\hline \multirow{2}{*}{ Fuste } & \multirow{2}{*}{$\begin{array}{l}\text { Comprimento Útil } \\
(\mathrm{m})\end{array}$} & \multirow{2}{*}{$\begin{array}{c}\text { Comprimento } \\
\text { Utilizado }^{1 /} \\
(\mathrm{m})\end{array}$} & \multicolumn{4}{|c|}{ Número de Toras } & \multirow{2}{*}{$\begin{array}{l}\text { Resíduo }^{2} \\
\text { (m) }\end{array}$} \\
\hline & & & $l_{1}=3,75 \mathrm{~m}$ & $l_{2}=4,35 \mathrm{~m}$ & $l_{3}=4,99 \mathrm{~m}$ & Total & \\
\hline 1 & 4,00 & 3,75 & 1 & 0 & 0 & 1 & 0,25 \\
\hline 2 & 4,50 & 4,35 & 0 & 1 & 0 & 1 & 0,15 \\
\hline 3 & 5,00 & 4,99 & 0 & 0 & 1 & 1 & 0,01 \\
\hline 4 & 5,50 & 4,99 & 0 & 0 & 1 & 1 & 0,51 \\
\hline 5 & 6,00 & 4,99 & 0 & 0 & 1 & 1 & 1,01 \\
\hline 6 & 6,50 & 4,99 & 0 & 0 & 1 & 1 & 1,51 \\
\hline 7 & 7,00 & 4,99 & 0 & 0 & 1 & 1 & 2,01 \\
\hline 8 & 7,50 & 7,50 & 2 & 0 & 0 & 2 & 0,00 \\
\hline 9 & 8,00 & 7,50 & 2 & 0 & 0 & 2 & 0,50 \\
\hline 10 & 8,50 & 8,10 & 1 & 1 & 0 & 2 & 0,40 \\
\hline 11 & 9,00 & 8,74 & 1 & 0 & 1 & 2 & 0,26 \\
\hline 12 & 9,50 & 9,34 & 0 & 1 & 1 & 2 & 0,16 \\
\hline 13 & 10,00 & 9,98 & 0 & 0 & 2 & 2 & 0,02 \\
\hline 14 & 10,50 & 9,98 & 0 & 0 & 2 & 2 & 0,52 \\
\hline 15 & 11,00 & 9,98 & 0 & 0 & 2 & 2 & 1,02 \\
\hline 16 & 11,50 & 11,25 & 3 & 0 & 0 & 3 & 0,25 \\
\hline 17 & 12,00 & 11,85 & 2 & 1 & 0 & 3 & 0,15 \\
\hline 18 & 12,50 & 12,49 & 2 & 0 & 1 & 3 & 0,01 \\
\hline 19 & 13,00 & 12,49 & 2 & 0 & 1 & 3 & 0,51 \\
\hline 20 & 13,50 & 13,09 & 1 & 1 & 1 & 3 & 0,41 \\
\hline 21 & 14,00 & 13,73 & 1 & 0 & 2 & 3 & 0,27 \\
\hline 22 & 14,50 & 14,33 & 0 & 1 & 2 & 3 & 0,17 \\
\hline 23 & 15,00 & 15,00 & 4 & 0 & 0 & 4 & 0,00 \\
\hline 24 & 15,50 & 15,00 & 4 & 0 & 0 & 4 & 0,50 \\
\hline 25 & 16,00 & 15,60 & 3 & 1 & 0 & 4 & 0,40 \\
\hline 26 & 16,50 & 16,24 & 3 & 0 & 1 & 4 & 0,26 \\
\hline 27 & 17,00 & 16,84 & 2 & 1 & 1 & 4 & 0,16 \\
\hline 28 & 17,50 & 17,48 & 2 & 0 & 2 & 4 & 0,02 \\
\hline 29 & 18,00 & 17,48 & 2 & 0 & 2 & 4 & 0,52 \\
\hline 30 & 18,50 & 18,08 & 1 & 1 & 2 & 4 & 0,42 \\
\hline 31 & 19,00 & 18,75 & 5 & 0 & 0 & 5 & 0,25 \\
\hline 32 & 19,50 & 19,35 & 4 & 1 & 0 & 5 & 0,15 \\
\hline 33 & 20,00 & 19,99 & 4 & 0 & 1 & 5 & 0,01 \\
\hline 34 & 20,50 & 19,99 & 4 & 0 & 1 & 5 & 0,51 \\
\hline 35 & 21,00 & 20,59 & 3 & 1 & 1 & 5 & 0,41 \\
\hline 36 & 21,50 & 21,23 & 3 & 0 & 2 & 5 & 0,27 \\
\hline 37 & 22,00 & 21,83 & 2 & 1 & 2 & 5 & 0,17 \\
\hline 38 & 22,50 & 22,50 & 6 & 0 & 0 & 6 & 0,00 \\
\hline 39 & 23,00 & 22,50 & 6 & 0 & 0 & 6 & 0,50 \\
\hline 40 & 23,50 & 23,10 & 5 & 1 & 0 & 6 & 0,40 \\
\hline 41 & 24,00 & 23,74 & 5 & 0 & 1 & 6 & 0,26 \\
\hline
\end{tabular}

1/ Soma dos comprimentos das toras retiradas.

2/ Diferença entre o comprimento utilizável e o comprimento utilizado. 
acrescentado o comprimento útil residual das toras com $d$ imediatamente anterior. Esta situação não foi considerada no exemplo apresentado.

No exemplo foram tratados os diferentes comprimentos de tora como se todos os produtos tivessem o mesmo preço unitário $\left(\mathrm{R} \$ / \mathrm{m}^{3}\right)$. No caso de se querer utilizar preços diferenciados por produto, além dos algoritmos descritos, deveriam ser utilizadas funções de afilamento ${ }^{1 /}$ para o cálculo dos volumes parciais correspondentes. Embora existam modelos de afilamento que permitem estimar diretamente as alturas em diferentes diâmetros comerciais, como é o caso das equações apresentadas em Demaershalk \& Kozak (1977), em se tratando dos polinômios de grau elevado deve ser utilizado algum algoritmo específico, como o método da bisseção, para obter as alturas nas quais se localizam os diferentes $d$ no fuste (Arce et al., 1999; Arce, 2000). É importante ressaltar que existem ainda sistemas de processamento de inventário florestal comercializados no Brasil que requerem do usuário o ajuste prévio de duas funções polinomiais de quinto grau independentes: uma para a estimativa de $d_{\mathrm{i}}=$ $f\left(h_{\mathrm{i}}\right)$ e outra para a estimativa de $h_{\mathrm{i}}=f\left(d_{\mathrm{i}}\right)$. As curvas que definem o perfil de uma mesma árvore a partir destes dois modelos, embora muito semelhantes, dificilmente apresentarão um ajuste perfeito.

\subsection{Comparação entre as Operações de Traçamento Real e Otimizado}

No Quadro 4 estão o resultado do traçamento realizado na floresta pelo motosserrista (situação real) e o resultado otimizado em nível de tora. Neste quadro é indicado o comprimento útil dos fustes medidos na floresta, a quantidade de toras obtidas em cada fuste de cada um dos três comprimentos utilizados, o comprimento total utilizado obtido através da soma dos comprimentos das toras e os resíduos de cada um dos fustes analisados.

Observa-se (Quadro 4) que em alguns casos os resíduos, obtidos como a diferença entre o comprimento útil até $d$ igual a $18 \mathrm{~cm}$ e o comprimento aproveitado pelo motosserrista, apresentam valores negativos, indicando que foi utilizado um comprimento superior ao indicado pelo $d$. Esta situação implica o fato de, provavelmente, algumas das toras obtidas possuírem um diâmetro em

\footnotetext{
1/ As funções de afilamento, funções de forma ou funções taper, estimam diâmetros a diferentes alturas $d_{i}=f\left(h_{i}\right)$.
}

ponta fina inferior ao estipulado para as toras para serraria $(18 \mathrm{~cm})$, podendo eventualmente ser rejeitadas pela indústria. Porém, por tratar-se de uma situação real de colheita, estes casos foram mantidos com a finalidade de caracterizar a situação real da melhor forma possível. Obviamente, padrões ótimos de corte nunca geraram estes resíduos negativos, uma vez que os dois algoritmos utilizados efetuam comparações de comprimentos até o comprimento máximo definido como útil.

No Quadro 4 observa-se que os padrões ótimos de corte não conseguem evitar por completo a geração de determinados resíduos nos fustes. Apenas para um único fuste, cujo comprimento útil foi de $11,85 \mathrm{~m}$, foi atingida a redução teórica completa deste resíduo, através do traçamento do mesmo em duas toras de $3,75 \mathrm{~m}$ e uma tora de 4,35 m ( 2 × 3,75 +4,35 = 11,85). Porém, o resíduo máximo segundo os padrões de corte otimizados foi de $2,41 \mathrm{~m}$, para um fuste com comprimento útil de 7,40 m. Paradoxalmente, neste caso o motosserrista conseguiu obter uma situação mais favorável, retirando duas toras de $3,75 \mathrm{~m}$ em vez de uma tora de $4,99 \mathrm{~m}$. O resíduo negativo obtido pelo motosserrista $(-0,18 \mathrm{~m})$ nunca poderia ter sido alcançado utilizando os padrões de corte, uma vez que os algoritmos 1 e 2 baseiam-se em critérios precisos de avaliação de possibilidades potenciais de corte. De qualquer forma, a habilidade do motosserrista em contornar situações difíceis, considerando que alguns fustes possuem defeitos como tortuosidade, bifurcações, dentre outros, é fundamental para atingir, conjuntamente com os padrões ótimos de corte, a maior eficiência nas operações de colheita florestal.

No Quadro 5 apresenta-se uma análise comparativa dos resíduos que efetivamente foram deixados na floresta após as tarefas de traçamento e os que poderiam ter sido deixados no caso de utilizar os padrões ótimos de corte.

Dos resíduos deixados na floresta foram considerados apenas os resíduos úteis, ou seja, aquelas toras cujo $d$ atingiu a exigência da indústria $(d \geq 18 \mathrm{~cm})$, mas por questões de comprimento foram abandonadas.

Observa-se no Quadro 5 que os mesmos comprimentos totais foram considerados para o conjunto de dados reais e otimizados. Isto foi possível devido ao fato de utilizar padrões ótimos de corte gerados, considerando intervalos de $1 \mathrm{~cm}$ no comprimento útil dos fustes. Neste sentido, o comprimento útil total, ou seja, a soma dos comprimentos úteis de todos os fustes, foi de $296,50 \mathrm{~m}$.

\section{R. Árvore, Viçosa-MG, v.28, n.2, p.207-217, 2004}


Quadro 4 - Resultados do traçamento real e do traçamento otimizado em nível de toras Table 4 -Results of real and optimized bucking operations at log level

\begin{tabular}{|c|c|c|c|c|c|c|c|c|c|c|c|c|c|}
\hline \multirow{3}{*}{ Fuste } & \multirow{3}{*}{$\begin{array}{l}\text { Comp. } \\
{\text { Útil }{ }^{1}}^{1 /} \\
\text { (m) }\end{array}$} & \multicolumn{6}{|c|}{ Traçamento Real } & \multicolumn{6}{|c|}{ Traçamento Otimizado } \\
\hline & & \multirow{2}{*}{$\begin{array}{c}\text { Comp. } \\
\text { usado } \\
(\mathrm{m})\end{array}$} & \multicolumn{4}{|c|}{ Número de toras ${ }^{2}$ - } & \multirow{2}{*}{$\begin{array}{l}\text { Resíduo } \\
(\mathrm{m})^{-3 /}\end{array}$} & \multirow{2}{*}{$\begin{array}{c}\text { Comp. } \\
\text { usado } \\
(\mathrm{m})\end{array}$} & \multicolumn{4}{|c|}{ Número de toras ${ }^{2}$} & \multirow{2}{*}{$\begin{array}{l}\text { Resíduo } \\
(\mathrm{m})^{3 /}\end{array}$} \\
\hline & & & $l_{1}$ & $l_{2}$ & $l_{3}$ & Total & & & $l_{1}$ & $l_{2}$ & $l_{3}$ & Total & \\
\hline 1 & 3,90 & 3,78 & 1 & 0 & 0 & 1 & 0,12 & 3,75 & 1 & 0 & 0 & 1 & 0,15 \\
\hline 2 & 5,50 & 3,80 & 1 & 0 & 0 & 1 & 1,70 & 4,99 & 0 & 0 & 1 & 1 & 0,51 \\
\hline 3 & 6,80 & 3,80 & 1 & 0 & 0 & 1 & 3,00 & 4,99 & 0 & 0 & 1 & 1 & 1,81 \\
\hline 4 & 6,85 & 7,60 & 2 & 0 & 0 & 2 & $-0,75$ & 4,99 & 0 & 0 & 1 & 1 & 1,86 \\
\hline 5 & 7,30 & 3,80 & 1 & 0 & 0 & 1 & 3,50 & 4,99 & 0 & 0 & 1 & 1 & 2,31 \\
\hline 6 & 7,40 & 7,58 & 2 & 0 & 0 & 2 & $-0,18$ & 4,99 & 0 & 0 & 1 & 1 & 2,41 \\
\hline 7 & 9,00 & 7,58 & 2 & 0 & 0 & 2 & 1,42 & 8,74 & 1 & 0 & 1 & 2 & 0,26 \\
\hline 8 & 9,03 & 7,58 & 2 & 0 & 0 & 2 & 1,45 & 8,74 & 1 & 0 & 1 & 2 & 0,29 \\
\hline 9 & 10,53 & 7,58 & 2 & 0 & 0 & 2 & 2,95 & 9,98 & 0 & 0 & 2 & 2 & 0,55 \\
\hline 10 & 10,77 & 7,57 & 2 & 0 & 0 & 2 & 3,20 & 9,98 & 0 & 0 & 2 & 2 & 0,79 \\
\hline 11 & 10,80 & 7,58 & 2 & 0 & 0 & 2 & 3,22 & 9,98 & 0 & 0 & 2 & 2 & 0,82 \\
\hline 12 & 11,50 & 8,12 & 1 & 1 & 0 & 2 & 3,38 & 11,25 & 3 & 0 & 0 & 3 & 0,25 \\
\hline 13 & 11,85 & 8,13 & 1 & 1 & 0 & 2 & 3,72 & 11,85 & 2 & 1 & 0 & 3 & 0,00 \\
\hline 14 & 11,90 & 11,96 & 2 & 1 & 0 & 3 & $-0,06$ & 11,85 & 2 & 1 & 0 & 3 & 0,05 \\
\hline 15 & 12,81 & 11,99 & 2 & 1 & 0 & 3 & 0,82 & 12,49 & 2 & 0 & 1 & 3 & 0,32 \\
\hline 16 & 13,86 & 13,24 & 1 & 1 & 1 & 3 & 0,62 & 13,73 & 1 & 0 & 2 & 3 & 0,13 \\
\hline 17 & 13,95 & 12,59 & 2 & 0 & 1 & 3 & 1,36 & 13,73 & 1 & 0 & 2 & 3 & 0,22 \\
\hline 18 & 14,30 & 11,90 & 2 & 1 & 0 & 3 & 2,40 & 13,73 & 1 & 0 & 2 & 3 & 0,57 \\
\hline 19 & 15,21 & 16,27 & 2 & 2 & 0 & 4 & $-1,06$ & 15,00 & 4 & 0 & 0 & 4 & 0,21 \\
\hline 20 & 16,12 & 13,70 & 0 & 2 & 1 & 3 & 2,42 & 15,60 & 3 & 1 & 0 & 4 & 0,52 \\
\hline 21 & 16,43 & 13,13 & 1 & 1 & 1 & 3 & 3,30 & 16,24 & 3 & 0 & 1 & 4 & 0,19 \\
\hline 22 & 16,90 & 13,70 & 0 & 2 & 1 & 3 & 3,20 & 16,84 & 2 & 1 & 1 & 4 & 0,06 \\
\hline 23 & 17,32 & 13,14 & 1 & 1 & 1 & 3 & 4,18 & 16,84 & 2 & 1 & 1 & 4 & 0,48 \\
\hline 24 & 18,15 & 14,34 & 0 & 1 & 2 & 3 & 3,81 & 18,08 & 1 & 1 & 2 & 4 & 0,07 \\
\hline 25 & 18,32 & 17,49 & 1 & 2 & 1 & 4 & 0,83 & 18,08 & 1 & 1 & 2 & 4 & 0,24 \\
\hline
\end{tabular}

1/ $L=$ Comprimento útil medido na floresta até um diâmetro com casca em ponta fina de $18 \mathrm{~cm}$.

2/ Número de toras por tipo de produto, segundo seu comprimento: $l_{1}=3,75 \mathrm{~m} ; l_{2}=4,35 \mathrm{~m} ; l_{3}=4,99 \mathrm{~m}$.

3/ Diferença entre o comprimento útil e o comprimento utilizado.

Analisando o fato de que em determinados fustes o resíduo deixado pelo motosserrista foi menor do que o resíduo sugerido pelos padrões ótimos de corte (fustes $4,6,14$ e 19), verifica-se que estas situações foram possíveis somente porque foi utilizado um comprimento útil maior que o efetivamente medido na floresta antes das operações de traçamento. O que deve ser aproveitado deste fato é a experiência prática do motosserrista, que em determinadas circunstâncias utiliza algum tipo de "tolerância intuitiva" para executar sua tarefa.

Sem considerar os resíduos negativos, em todos os demais casos os resíduos sugeridos pelos padrões ótimos de corte foram menores que os efetivamente obtidos na floresta. Enquanto o conjunto de dados reais apresentou um resíduo total de 48,55 m, com os padrões ótimos de corte este resíduo foi reduzido a $15,07 \mathrm{~m}$, o que equivale a um aumento no comprimento total utilizado de 247,95 a $281,43 \mathrm{~m}$.

A Figura 3 apresenta o número de casos observados por classe de magnitude do resíduo. Observa-se que os resíduos otimizados distribuem-se predominantemente nas primeiras duas classes, com até 5 e $10 \%$ do comprimento útil desperdiçado, respectivamente, enquanto os resíduos reais apresentam números de casos observados 


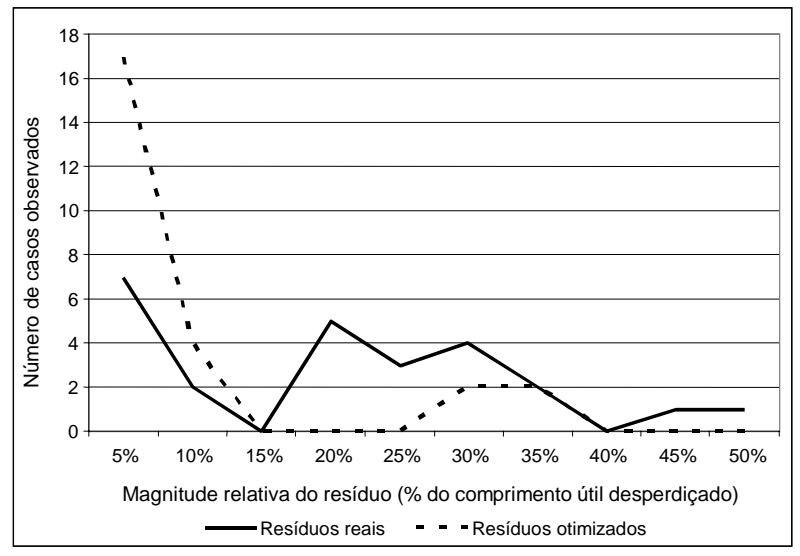

Figura 3 - Contagem dos resíduos por classe de magnitude relativa $(\%)$.

Figure 3 - Residue count by relative magnitude classes (\%). mais elevados nas classes de $20,25,30,45$ e $50 \%$ do comprimento útil desperdiçado.

Em termos relativos, o Quadro 5 mostra que o resíduo deixado na floresta, equivalente a $16 \%$ do comprimento útil total, foi reduzido para 5\% com a utilização dos padrões ótimos de corte. Estes números ilustram o ganho potencial de utilizar este tipo de procedimento próprio da Pesquisa Operacional nas atividades florestais de traçamento de fustes.

A experiência prática do motosserrista, que o possibilitou utilizar certos níveis de tolerância nos comprimentos e nos diâmetros em ponta fina, deveria ser incorporada de alguma maneira neste tipo de soluções matemáticas, visando aprimorar ainda mais a eficiência das operações florestais de colheita.

Quadro 5 - Comparação entre o traçamento real e otimizado

Table 5 - Comparison between real and optimized bucking operations

\begin{tabular}{|c|c|c|c|c|c|c|c|c|}
\hline \multirow{4}{*}{ Fuste } & \multicolumn{4}{|c|}{ Dados Reais } & \multicolumn{4}{|c|}{ Dados Otimizados } \\
\hline & \multicolumn{2}{|c|}{ Comprimento } & \multicolumn{2}{|c|}{ Resíduo } & \multicolumn{2}{|c|}{ Comprimento } & \multicolumn{2}{|c|}{ Resíduo } \\
\hline & Total & Utilizado & Absoluto & Relativo & Total & Utilizado & Absoluto & Relativo \\
\hline & $(\mathrm{m})$ & $(\mathrm{m})$ & $(\mathrm{m})$ & $\%$ & $(\mathrm{~m})$ & (m) & $(\mathrm{m})$ & $\%$ \\
\hline 1 & 3,90 & 3,78 & 0,12 & 3,08 & 3,90 & 3,75 & 0,15 & 3,85 \\
\hline 2 & 5,50 & 3,80 & 1,70 & 30,91 & 5,50 & 4,99 & 0,51 & 9,27 \\
\hline 3 & 6,80 & 3,80 & 3,00 & 44,12 & 6,80 & 4,99 & 1,81 & 26,62 \\
\hline 4 & 6,85 & 7,60 & $-0,75$ & $-10,95$ & 6,85 & 4,99 & 1,86 & 27,15 \\
\hline 5 & 7,30 & 3,80 & 3,50 & 47,95 & 7,30 & 4,99 & 2,31 & 31,64 \\
\hline 6 & 7,40 & 7,58 & $-0,18$ & $-2,43$ & 7,40 & 4,99 & 2,41 & 32,57 \\
\hline 7 & 9,00 & 7,58 & 1,42 & 15,78 & 9,00 & 8,74 & 0,26 & 2,89 \\
\hline 8 & 9,03 & 7,58 & 1,45 & 16,06 & 9,03 & 8,74 & 0,29 & 3,21 \\
\hline 9 & 10,53 & 7,58 & 2,95 & 28,02 & 10,53 & 9,98 & 0,55 & 5,22 \\
\hline 10 & 10,77 & 7,57 & 3,20 & 29,71 & 10,77 & 9,98 & 0,79 & 7,34 \\
\hline 11 & 10,80 & 7,58 & 3,22 & 29,81 & 10,80 & 9,98 & 0,82 & 7,59 \\
\hline 12 & 11,50 & 8,12 & 3,38 & 29,39 & 11,50 & 11,25 & 0,25 & 2,17 \\
\hline 13 & 11,85 & 8,13 & 3,72 & 31,39 & 11,85 & 11,85 & 0,00 & 0,00 \\
\hline 14 & 11,90 & 11,96 & $-0,06$ & $-0,50$ & 11,90 & 11,85 & 0,05 & 0,42 \\
\hline 15 & 12,81 & 11,99 & 0,82 & 6,40 & 12,81 & 12,49 & 0,32 & 2,50 \\
\hline 16 & 13,86 & 13,24 & 0,62 & 4,47 & 13,86 & 13,73 & 0,13 & 0,94 \\
\hline 17 & 13,95 & 12,59 & 1,36 & 9,75 & 13,95 & 13,73 & 0,22 & 1,58 \\
\hline 18 & 14,30 & 11,90 & 2,40 & 16,78 & 14,30 & 13,73 & 0,57 & 3,99 \\
\hline 19 & 15,21 & 16,27 & $-1,06$ & $-6,97$ & 15,21 & 15,00 & 0,21 & 1,38 \\
\hline 20 & 16,12 & 13,70 & 2,42 & 15,01 & 16,12 & 15,60 & 0,52 & 3,23 \\
\hline 21 & 16,43 & 13,13 & 3,30 & 20,09 & 16,43 & 16,24 & 0,19 & 1,16 \\
\hline 22 & 16,90 & 13,70 & 3,20 & 18,93 & 16,90 & 16,84 & 0,06 & 0,36 \\
\hline 23 & 17,32 & 13,14 & 4,18 & 24,13 & 17,32 & 16,84 & 0,48 & 2,77 \\
\hline 24 & 18,15 & 14,34 & 3,81 & 20,99 & 18,15 & 18,08 & 0,07 & 0,39 \\
\hline 25 & 18,32 & 17,49 & 0,83 & 4,53 & 18,32 & 18,08 & 0,24 & 1,31 \\
\hline Total & 296,50 & 247,95 & 48,55 & 16,37 & 296,50 & 281,43 & 15,07 & 5,08 \\
\hline
\end{tabular}

\section{R. Árvore, Viçosa-MG, v.28, n.2, p.207-217, 2004}




\section{CONCLUSÃO}

A utilização de técnicas de Pesquisa Operacional foi eficaz para obtenção de padrões de corte ótimos para fustes de árvores comerciais. O algoritmo de geração de números úteis reduziu o número potencial de cortes que devem ser avaliados ao longo do comprimento do fuste. O algoritmo de programação dinâmica garante a obtenção de padrões ótimos de corte, considerando os fustes existentes e os comprimentos de tora dos produtos demandados pela indústria.

A utilização dos padrões ótimos de corte permitiu redução nos resíduos reais, equivalentes a 16\% do comprimento total utilizado, para $5 \%$, considerando os resíduos teóricos.

\section{REFERÊNCIASBIBLIOGRÁFICAS}

ANDRADE, V. C. L.; LEITE, H. G. Um método para quantificar multiprodutos de árvores individuais na unidade estere. Revista Árvore, v. 22, n. 3, p. 299-306, 1998.

ARCE, J. E. Um sistema de análise, simulação e otimização do sortimento florestal em função da demanda por multiprodutos e dos custos de transporte. 2000. 129 f. Tese (Doutorado em Ciências Florestais) Universidade Federal do Paraná, Curitiba, 2000.

ARCE, J. E. et al. Patrones individuales óptimos de corte para la obtención de multiproductos forestales. In: CONGRESO FORESTAL LATINOAMERICANO, 1., 1999, Lima, Peru. Anais... Lima, Perú: 1999. (CD ROM Abstract).

ARENALES, M. N.; MORABITO, R. O problema de corte e empacotamento e aplicações industriais. In: CONGRESSO NACIONAL DE MATEMÁTICA APLICADA E COMPUTACIONAL, 2., OFICINA NACIONAL DE PCE, 2., 1997, Gramado-RS. Anais... Gramado: 1997. 146 p.

ASSIS, A. L. et al. Comparação de modelos polinomiais segmentados e não-segmentados na estimativa de diâmetros e volumes ao longo do fuste de Pinus taeda. Cerne, v. 7, n. 1, p. 20-40, 2001.

BORGES J. F. Seccionamento do fuste de Pinus taeda $\mathbf{L}$. para obtenção do volume de madeira serrada através da função de forma polinomial. 1981. $81 \mathrm{f}$. Dissertação (Mestrado em Ciências Florestais) - Universidade Federal do Paraná, Curitiba, 1981.

CARNIERI, C.; MENDOZA, G. A.; GAVINHO, L. G. Solution procedures for cutting lumber into furniture parts. European Journal of Operational Research, v. 73, p. 495-501, 1994a.
CARNIERI, C.; MENDOZA, G. A.; GAVINHO, L. G. Optimal cutting of lumber and particleboards into dimension parts: some algorithms and solution procedures. Wood and Fiber Science, v. 26, n. 1, p. 131-141, 1994b.

DEADMAN, M. W.; GOULDING, C. J. A method for assessment of recoverable volume by log types. New Zeland Forest of Science, v. 9, n. 2, p. 225-239, 1978.

DEMAERSHALK, J. P.; KOZAK A. The whole bole system: a conditioned dual-equation system for precise prediction of tree profiles. Canadian Journal Forest of Research, v. 7, p. 488-497, 1977.

EPSTEIN R. et al. Use of OR Systems in the Chilean Forest Industries. Interfaces, v. 29, n. 1, p. 7-29, 1999.

FAALAND, B.; BRIGGS, D. Log bucking and lumber manufacturing using dynamic programming. Management Science, v. 30, n. 2, p. 245-257, 1984.

GILMORE, P. C.; GOMORY R. E. A linear programming approach to the cutting stock problem. Operations Research, v. 9, p. 848-859, 1961.

GILMORE, P. C.; GOMORY R. E. A linear programming approach to the cutting stock problem, Part II. Operations Research, v. 11, p. 863-888, 1963.

GILMORE, P. C.; GOMORY R. E. Multi-stage stock cutting problems of two or more dimensions. Operations Research, v. 13, p. 94-120, 1965.

GREGERSEN, H.; CONTRERAS A. Análisis económico de proyectos forestales. Roma: FAO Montes, 1980.

JORGE, L. A. B. Utilização de curvas de forma em séries relativas transformadas nas estimativas de volumes totais e parciais de árvores de Pinus taeda $\mathbf{L}$. 1982. 91 f. Dissertação (Mestrado em Ciências Florestais) - Universidade Federal do Paraná, Curitiba, 1982.

MANESS T. C.; ADAMS D. A. The combined optimization of log bucking and sawing strategies. Wood and Fiber Science, v. 23, n. 2, p. 296-314, 1991.

OLIVEIRA, E. B. Um sistema computadorizado de prognose do crescimento e produção de Pinus taeda L., com critérios quantitativos para a avaliação técnica e econômica de regimes de manejo. 1995. $134 \mathrm{f}$. Tese (Doutorado em Ciências Florestais) - Universidade Federal do Paraná, Curitiba, 1995.

REINDERS, M. P.; HENDRIKS, Th. H. B. Lumber production optimization. European Journal of Operational Research, v. 42, p. 243-253, 1989. 